\title{
COVID-19 \& Ciência: O valor do conhecimento em tempos de pandemia
}

\author{
COVID-19 and Science: The value of knowledge in times of pandemic \\ COVID-19 y ciencia: EI valor del conocimiento en tiempos de pandemia
}

Recebido: 16/04/2021 | Revisado: 25/04/2021 | Aceito: 29/04/2021 | Publicado: 14/05/2021

\author{
Iukary Takenami \\ ORCID: https://orcid.org/0000-0001-5660-7766 \\ Universidade Federal do Vale do São Francisco, Brasil \\ E-mail: iukary.takenami@univasf.edu.br \\ Maria Augusta Vasconcelos Palácio \\ ORCID: https://orcid.org/0000-0002-2780-125X \\ Universidade Federal do Vale do São Francisco, Brasil \\ E-mail: augusta.palacio@univasf.edu.br \\ Pablo Rafael Silveira Oliveira \\ ORCID: https://orcid.org/0000-0001-9541-3737 \\ Universidade Federal da Bahia, Brasil \\ E-mail: prsoliveira@ufba.br
}

\begin{abstract}
Resumo
A doença do coronavírus 2019 (COVID-19) tornou-se uma pandemia alarmante para o mundo e, desde a sua primeira identificação, evocou nos chefes de estados e na população em geral rápidas respostas para o seu enfretamento. Diante desse cenário de pandemia global, a ciência (re)emergiu como uma importante ferramenta de apoio, alcançando maior visibilidade e projeção na sociedade. Considerando a importância da ciência como uma forma de produzir conhecimento sistemático e confiável, esse estudo propõe-se a apresentar observações na forma de um ensaio teóricoreflexivo construído por meio da análise da literatura publicada em livros e artigos científicos acerca do papel da ciência no contexto da COVID-19, tendo em vista a prática científica, os caminhos percorridos, os avanços tecnológicos, os entraves, os percursos possíveis e sua relação com a formação da cidadania na sociedade. Diante de tantos estudos publicados na literatura, torna-se importante discutir e amadurecer os achados científicos mediante análise crítica-reflexiva de uma coletividade de indivíduos capazes de realizar um julgamento fundamentado. Ademais, o fomento à educação e o letramento científico são imprescindíveis no desenvolvimento da autonomia e criticidade da sociedade. Do contrário, interpretações equivocadas e falácias pseudocientíficas podem interferir negativamente na governança, gestão em saúde e na sociedade, sugerindo políticas públicas inconsistentes e colocando em risco à saúde da população.
\end{abstract}

Palavras-chave: COVID-19; Pandemia; Ciência; Conhecimento científico.

\begin{abstract}
Coronavirus disease 2019 (COVID-19) has become a global pandemic and, since its first identification, evoked in the heads of states and the general population faster responses to cope with it. Faced with this global pandemic scenario, science (re)emerged as an important support tool, achieving greater visibility and projection in society. Considering the importance of science to produce systematic and reliable knowledge, this study proposes to present observations in the form of a theoretical-reflective essay constructed by analyzing the literature published in books and scientific articles about the role of science in the context of COVID-19, in view of scientific practice, the paths technological advances, obstacles, possible paths and their relationship with the formation of citizenship in society. In view of so many studies published in the literature, it is important to discuss and mature scientific findings through a criticalreflexive analysis by individuals within a group capable of making a well-founded judgment. In addition, the promotion of education and scientific literacy are essential in the development of society's autonomy and criticality. Otherwise, misinterpretations and pseudoscience fallacies may negatively effects on governance, health management and society, suggesting inconsistent public policies and putting the population's health at risk.
\end{abstract}

Keywords: COVID-19; Pandemic; Science; Scientific knowledge.

\section{Resumen}

La enfermedad del coronavirus 2019 (COVID-19) se ha convertido en una pandemia alarmante para el mundo y, desde su primera identificación, evocó en los jefes de estado y en la población en general respuestas rápidas para hacerle frente. Ante este escenario de pandemia global, la ciencia (re)emergió como una importante herramienta de apoyo, logrando una mayor visibilidad y proyección en la sociedad. Considerando la importancia de la ciencia como forma de producir conocimiento sistemático y confiable, este estudio propone presentar observaciones en forma de ensayo teórico-reflexivo construido a partir del análisis de la literatura publicada en libros y artículos científicos sobre 
el papel de la ciencia en el contexto de COVID-19, en vista de la práctica científica, los caminos tecnológicos avances, obstáculos, posibles caminos y su relación con la formación de la ciudadanía en la sociedad. Ante tantos estudios publicados en la literatura, es importante discutir y madurar los hallazgos científicos a través de un análisis crítico-reflexivo de un grupo de individuos capaces de emitir un juicio informado. Además, la promoción de la educación y la alfabetización científica son fundamentales para el desarrollo de la autonomía y la criticidad de la sociedad. De lo contrario, las malas interpretaciones y las falacias pseudocientíficas pueden interferir negativamente con la gobernabilidad, la gestión de la salud y la sociedad, sugiriendo políticas públicas inconsistentes y poniendo en riesgo la salud de la población.

Palabras-clave: COVID-19; Pandemia; Ciencia; Conocimiento científico.

\section{Introdução}

A doença do coronavírus 2019 (COVID-19) é um dos maiores desafios globais dos últimos cem anos. Os primeiros relatos da doença surgiram em dezembro de 2019, no município de Wuhan, província de Hubei, China. Logo em seguida, foi mostrado que se tratava de uma nova cepa de betacoronavírus (CoV), batizada de SARS-CoV-2, causadora de síndrome respiratória aguda grave (SARS) em humanos (Wu et al., 2020). Em 11 de março de 2020, a Organização Mundial da Saúde (OMS) declarou estado de pandemia para a COVID-19 (WHO, 2020a). Na época, a propagação exponencial do vírus e os seus impactos sociais, econômicos e políticos estimularam rápidas respostas à COVID-19 por parte de chefes de estado, OMS, instituições de saúde e população em geral. Desde então, a OMS tem disponibilizado inúmeras atualizações epidemiológicas e operacionais no seu site oficial, realizado múltiplas conferências com o objetivo de divulgar os dados epidemiológicos a nível mundial e impulsionado o intercâmbio global de boas práticas, desafios, enfoques inovadores e estratégicas efetivas para auxiliar na tomada de decisões (WHO, 2020b, WHO, 2020c), sempre fomentada por meio do uso de evidências científicas de qualidade e acessíveis.

Hoje, um pouco mais de um ano após a identificação dos primeiros casos da COVID-19 em Wuhan, a "ciência" tornou-se grande protagonista do atual cenário mundial, pautando debates políticos, sociais e econômicos. Não se pode negar que a pandemia da COVID-19 despertou um interesse maior da sociedade civil pela "ciência", permitindo revisitar a afirmação de Alan Chalmers (1993, p.17), de que "nos tempos modernos, a ciência é altamente considerada”. Para o autor:

Há uma crença amplamente aceita de que há algo especial a respeito da ciência e de seus métodos. A atribuição do termo "científico" a alguma afirmação, linha de raciocínio ou peça de pesquisa é feita de um modo que pretende implicar algum tipo de mérito ou um tipo especial de confiabilidade (Chalmers, 1993, p.17).

Todavia, desde os primórdios da história, essa prática é reconhecida como uma das principais fontes de conhecimento e dinamização da sociedade, avançando substancialmente no padrão e na qualidade de vida da população (Novaes; Carvalheiro, 2007). No entanto, mais recentemente, a pandemia da COVID-19 despertou, especialmente no público leigo, um novo olhar para a ciência, revelando um processo de construção do conhecimento científico que nem sempre é linear e acumulativo.

$\mathrm{Na}$ prática, observa-se que muitas políticas públicas e ações foram elaboradas e implementadas em diferentes contextos e em graus variados pelo poder público, mas com base em pesquisas científicas conduzidas com rigor metodológico. O uso obrigatório de máscaras de proteção facial, o fechamento de estabelecimentos não essenciais e fronteiras aéreas, o cancelamento de eventos sociais e atividades públicas, foram apenas algumas das inúmeras medidas adotadas com o objetivo de evitar aglomerações, novos focos de contágio e preservar a distância mínima recomendada entre pessoas (Jones et al., 2020). Contudo, a resposta de enfrentamento à COVID-19 nem sempre tem sido alinhada aos critérios técnicos e científicos disponíveis.

No início da pandemia, e ainda hoje, muitas autoridades do campo político ainda refutam ou minimizam os efeitos do vírus, negando as medidas de distanciamento físico na redução do número de casos da doença e defendendo o uso de 
medicamentos sem eficácia comprovada ou, comprovadamente, ineficazes para o tratamento da COVID-19, entre outros. Cenários que têm contribuído para a propagação do vírus, que já ultrapassa a marca dos 128 milhões de casos diagnosticados de infecção e 2 milhões óbitos ligados à COVID-19, com uma taxa de letalidade estimada em 2,2\% (JHU, 2021). No Brasil, a flexibilização das medidas de isolamento social, notadamente após o período do carnaval em fevereiro de 2021, tem proporcionado a re(circulação) do SARS-CoV-2, gerando uma segunda onda de contágio com quantitativo de casos ainda maior do que no início da pandemia (Inloco, 2021). Não obstante, permeando todo esse cenário, autoridades e profissionais de saúde que estão na linha de frente também encontram dificuldades na educação da população em seguir as recomendações dos órgãos oficiais de saúde (Palácio; Takenami, 2020).

É importante salientar que a grande capacidade de disseminação do SARS-CoV-2, inclusive a partir de indivíduos assintomáticos (Gandhi; Yokoe; Havlir, 2020), associado à ausência de imunidade populacional prévia (por tratar-se de uma doença causada por uma nova cepa de CoV), e à inexistência de alternativas profiláticas eficazes contra a COVID-19, tornam o seu controle muito mais difícil. Soma-se a esses fatores a vulnerabilidade de grande parcela da população brasileira, evidenciada pela desigualdade de acesso a testes, atendimento médico e pela prevalência de condições de saúde como obesidade, cardiopatias, diabetes, entre outras (Brasil, 2020a). Se por um lado, muitos aspectos contribuem para o avanço da pandemia e o aumento da taxa de óbito, por outro, o trabalho colaborativo de vários grupos de pesquisa, o desenvolvimento de protocolos terapêuticos eficazes para a COVID-19, a melhor compreensão sobre a imunopatologia e o desenvolvimento de imunizantes em tempo recorde, a exemplo das vacinas, AstraZeneca, CoronaVac, Pfizer-BioNTech, Sinopharm e Sputinik, são alguns exemplos de avanços significativos na área.

Ainda que as vacinas sejam testadas em ensaios clínicos prévios, a sua real eficácia só poderá ser determinada após a aplicação de doses em larga escala. Assim, as clássicas medidas de saúde pública e vigilância como higienização das mãos e distanciamento físico, ainda representam as melhores estratégias para controlar a disseminação do SARS-CoV-2 (WHO, 2020d, Wilder-Smith; Freedman, 2020). Considerando a ciência como uma importante forma de produzir conhecimento sistemático e confiável, esse estudo se propôs a realizar um ensaio teórico-reflexivo acerca do papel da ciência no contexto da COVID-19, tendo em vista a prática científica, os caminhos percorridos, os avanços tecnológicos, os entraves, os percursos possíveis e sua relação com a formação da cidadania na sociedade atual.

\section{Metodologia}

Trata-se de um ensaio teórico-reflexivo construído a partir da análise da literatura publicada em livros e artigos científicos acerca do papel da ciência e suas repercussões no contexto da COVID-19. Segundo Meneghetti (2011), um ensaio crítico caracteriza-se pela sua natureza reflexiva e interpretativa para entender uma realidade, um fenômeno ou uma situação específica.

Assim, artigos científicos sobre a COVID-19 foram pesquisados nas bases de dados Medical Literature Analysis and Retrieval System Online via Public/Publisher Medline (MedLine/PubMed) e Scientific ElectronicLibrary Online (SciELO), utilizando os seguintes descritores em português: "COVID-19", "SARS-CoV-2" e "pandemia", e seus respectivos em inglês: “COVID-19", "SARS-CoV-2" e "pandemic", no período de janeiro de 2020 a março de 2021. Em seguida, a busca de textos significativos sobre a ciência, metodologia científica, métodos e técnicas de pesquisa, publicação e qualidade científica foram pesquisados em livros e artigos científicos, sem período de tempo definido, considerando literaturas clássicas da área e referências que trazem perspectivas atualizadas sobre "ciência" e "conhecimento científico" .

Após a busca e leitura minuciosa dos textos de livros/artigos, selecionaram-se os conteúdos pertinentes para a reflexão sobre esta temática, e com isso, foram elaborados três tópicos norteadores para apoiar o ensaio teórico e a reflexão do estudo: 
"Um novo olhar para um velho fenômeno - a ciência", "Ciência, tecnologia e informação: os impactos negativos da desinformação" e, por fim, "A ciência em tempos de crise: entraves e caminhos possíveis".

\section{Um Novo Olhar Para um Velho Fenômeno - A Ciência.}

Em termos etimológicos 'ciência' é uma palavra derivada do latim, scientia, que significa conhecimento (Leite, 2008). Na visão de Bynun (2018), a ciência foi usada em conjunto com magia, religião e tecnologia pelas primeiras sociedades humanas que se estabeleceram em vales fluviais na Índia, na China e no Oriente Médio. Por outro lado, Visoni e Canalle (2009) relatam que os primeiros cientistas eram sacerdotes, a exemplo do primeiro cientista brasileiro, Bartolomeu Lourenço de Gusmão. Para Chalmers:

Francis Bacon foi um dos primeiros a tentar articular o que é o método da ciência moderna. No início do século XVII, propôs que a meta da ciência é a melhoria da vida do homem na terra e, para ele, essa meta seria alcançada através da coleta de fatos com observação organizada e derivando teorias a partir daí (Chalmers, 1993, p.20).

E assim, ao longo da história, a ciência passou por mudanças e transformações que influenciaram no modo como ela se encontra estruturada hoje. As concepções mais modernas de ciência consideram que a objetividade atribuída a esse tipo de conhecimento deve levar em consideração que não há 'um método científico' único e que o conhecimento científico é produzido a partir de uma grande diversidade de estratégias. Atualmente, a ciência pode ser entendida como um conjunto de conhecimentos específicos e sistematizados, com métodos, técnicas e objetos próprios. Fatos que consolidaram a ciência como a maior comunidade epistêmica de prestígio e de credibilidade dos séculos XIX e XX, disseminando uma corrente positivista na qual o saber científico era a única forma de conhecimento verdadeiro (Oliveira, 2020). Embora existam conhecimentos provenientes do empirismo, religião e filosofia, didaticamente, somente o conhecimento científico, alicerçado na razão e objetividade, recebe o nome de ciência (Leite, 2008).

Ao contrário do que a grande maioria dos indivíduos imagina, "fazer ciência" não se trata apenas de microscópio, tubos de ensaios ou jaleco branco, mas de uma ampla gama de ferramentas metodológicas que permitem responder à curiosidade do ser humano. Cabe aqui destacar que as atividades desenvolvidas em laboratório são apenas uma das formas de fazer ciência. A propósito da citação de Francelin (2004, p.27), “a ciência não se reduz a experimentos, pelo contrário, é extremamente abrangente e complexa". Portanto, é por meio das diversas formas de fazer ciência que a mesma progride e atinge seus objetivos, de servir como instrumento de desenvolvimento do ser humano e da sua sociedade.

Para Volpato (2008), um dos principais motores para o avanço da ciência é a curiosidade. A curiosidade representa o cerne dessa prática e, para tanto, impulsiona o ser humano a fazer perguntas desde as suas origens. Em tempos de pandemia, a busca por respostas tem aguçado a curiosidade dos pesquisadores e da população em geral. Como surgiu o SARS-CoV-2? Como o vírus é transmitido? Indivíduos infectados e assintomáticos transmitem a doença? Quais mecanismos imunopatológicos estariam envolvidos na maior taxa de letalidade encontrada nos indivíduos pertencentes aos grupos de risco? A infecção por SARS-CoV-2 gera imunidade duradoura? Há possibilidade de sequelas após a alta?

$\mathrm{O}$ interesse da sociedade em questões científicas mostra que as discussões científicas não devem se restritas a especialistas. No entanto, são eles, pesquisadores ou cientistas, que lançam mão de diferentes métodos (metodologias científicas) para tentar estabelecer, por exemplo, relações de causa (exposição) e efeito (desfecho) para os fenômenos observados. Até o presente momento, a aplicação de métodos científicos tem permitido responder os questionamentos acima e compreender melhor os diversos aspectos da COVID-19, tornando factível, em um curto espaço de tempo, o desenvolvimento e aprovação de alguns tipos de imunizantes. A despeito da relação causal, diversos estudos têm observado maior taxa de óbito (efeito/desfecho) em indivíduos que, além da infecção por SARS-CoV-2, apresentam idade acima de 65 anos, hipertensão 
arterial ou diabetes mellitus (causas/exposição). Enquanto gestantes, puérperas e/ou na faixa pediátrica abaixo de cinco anos apresentam descompensação ou até agravamento dos sintomas respiratórios da COVID-19 (Brasil, 2020b). Ou ainda, apesar de não estarem totalmente elucidados, alguns estudos já têm demonstrado casos de reinfecção, sugerindo que a infeção por SARSCoV-2 pode não induzir uma imunidade duradoura (Selvaraj; Herman; Dapaah-Afriyie, 2020).

Nesse contexto, ressalta-se que os resultados de experimentos e/ou observações isoladas, não representam em si o conhecimento científico. Como fonte de conhecimento considerado confiável, este deve emergir da obtenção de múltiplas evidências e de um processo contínuo de críticas realizadas por pesquisadores treinados. A filósofa e historiadora da ciência, Naomi Oreskes (2019), afirma que os fatos científicos são afirmações às quais os cientistas estão de acordo. Assim, para ganhar robustez científica é necessário replicar o estudo e obter os mesmos resultados em diversos contextos. Trata-se, segundo Dal-Fabbro (2011, p.295) da analogia, "se muitas linhas de investigação levam à mesma conclusão então, a evidência de relação causal ficará mais forte”.

Entende-se também que os pesquisadores desempenham papel de contestar determinado conhecimento, isto é, refutar as assertivas científicas caso as mesmas sejam inconsistentes em relação a uma hipótese testada. Segundo Popper (1972), a ciência avança de acordo com o que ele chamou de conjecturas e refutações entre teorias contrárias, trazendo à luz informações que passaram despercebidas, que foram equivocadamente interpretadas ou hipóteses que expliquem melhor os fenômenos observados. Desse modo, o conhecimento gerado pela ciência pode sofrer mudanças, tornando-a dinâmica e, constantemente, em evolução. O conhecimento ou informação disponível hoje, pode não ser mais validado ou reconhecido amanhã, isso ocorre porque o conhecimento científico não é absoluto e definitivo, mas criticável em uma perspectiva realista, podendo mudar, por exemplo, conforme o ambiente, sujeito e relações circunstanciais.

Artigos científicos podem gerar, eventualmente, informações convenientes, mas que não necessariamente representam uma verdade científica. No início da pandemia, um estudo realizado na França com apenas 36 pacientes internados com a COVID-19 sugeriu que o uso da hidroxicloroquina associada à azitromicina poderia zerar a carga viral dos pacientes em seis dias (Gautret et al., 2020). Esse estudo promoveu, juntamente, com o posicionamento público de líderes mundiais, uma corrida pela compra da cloroquina e sua variante, a hidroxicloroquina, em um curto espaço de tempo, tornando o medicamento escasso para aqueles que já faziam uso contínuo da droga. Na época, os achados foram refutados a partir de investigações mais robustas e consistentes, demonstrando que não existem benefícios suficientes que justifiquem seu uso em pacientes diagnosticados com a COVID-19, em qualquer que seja a fase da doença (Mahevas et al., 2020; Tang et al., 2020). Hoje, os pesquisadores são categóricos ao afirmar que a cloroquina ou a hidroxicloroquina não tem efeito no tratamento da COVID-19, e que efeitos colaterais (como arritmia cardíaca) têm sido observados nos pacientes que fizeram uso desses fármacos (Mahevas et al., 2020; Tang et al., 2020).

Os resultados obtidos por meio das pesquisas científicas, utilizando métodos próprios e sistematizados, são publicados nos chamados periódicos científicos, classificados por áreas de conhecimento e por abrangência nacional ou internacional. A classificação por área de conhecimento facilita o encontro com o público que se deseja atingir e, assim, o periódico assume o papel de principal veículo formal da comunicação científica. A prática de tornar público e defender as conclusões dos estudos visa a construção de um conhecimento que constantemente se corrige. Segundo Volpato (2008, p.2), “o conhecimento, enquanto individual, pode satisfazer curiosidades pessoais. Mas, se permanecer no nível individual será levado ao túmulo e de nada valeu para essa sociedade que direta, ou indiretamente, o sustentou". Quando o conhecimento chega a outros cientistas, ele é visto e criticado, o que o enriquece e aprimora os saberes e as práticas. Além disso, uma vez publicado, pode ser elemento chave para que outros cientistas tenham novos insights ou alicercem suas novas criações. Fomentados pela tecnologia, esse ambiente social de troca de informações faz crescer rapidamente o potencial de conhecimento sobre a COVID-19, mas também de estudos com potencial conflitos de interesse. 
A leitura de um artigo científico, fenômeno traduzido em palavras da ciência feita por um determinado grupo de pesquisadores, deve ser eminentemente crítica-reflexiva. Durante esse processo é razoável avaliar, segundo critérios rigorosos, a qualidade de artigos científicos. No entanto, segundo Szklo (2006), não há como julgar a qualidade de um artigo científico, visto que não há "fatores de risco" para um artigo sem qualidade. Por outro lado, refletir e analisar criticamente é a melhor forma de fazer uma análise justa e plural. Para isso, cabe aos pesquisadores, profissionais que foram formados nesse campo, analisar criticamente, ponderar e mostrar para o público leigo o processo de construção do conhecimento científico. Assim, a produção científica está associada à divulgação como etapa de um processo atribuída ao pesquisador, realçando a função social de sua atividade profissional e fortalecendo uma inter-relação dinâmica e interativa entre ciência e sociedade (Conceição; Chagas, 2020; Dantas; Deccache-Maia, 2020).

A produção científica no último ano ganhou uma proporção nunca vista em outros períodos. Na base de dados PubMed/MEDLINE, utilizando o descritor "COVID-19”, até o dia $1^{\circ}$ de abril de 2021, foram publicados 118.397 artigos, aproximadamente, 263 artigos por dia. Para além dos artigos científicos já publicados, os preprints, relatórios preliminares de trabalho que não foram certificados pela revisão por pares, têm se tornado populares pela rapidez de compartilhamento de informações e como forma de obter sugestões e críticas de cientistas e não cientistas. Por outro lado, por mais que se configurem como informações preliminares que podem contribuir com o conhecimento que está sendo construído em determinada área, conforme Sheldon (2018), os preprints podem promover confusão e distorção dos dados científicos, de tal forma que um trabalho fraco, que não foi revisado, pode ganhar grandes proporções midiáticas, gerando desinformação e fomentando informações pseudocientíficas, o que dificulta a elaboração e o estabelecimento de estratégias mais condizentes com o conhecimento científico.

Um exemplo dessa necessidade de revisões mais críticas sobre os preprints refere-se ao estudo francês que recomendava a hidroxicloroquina para o tratamento da COVID-19. Após duras críticas pela comunidade científica, os próprios autores decidiram por apagá-lo de uma base de preprints. Mais recentemente, um trabalho publicado sobre um surto da COVID-19, como consequência do consumo de supostos animais chamados de 'Zubats' (referência a um desenho animado chamado Pokémon), mostrou que o referido periódico de publicação é suspeito de ser uma revista "predatória" (Elm et al., 2020). Segundo Beall (2017), os periódicos científicos predatórios são baseados em falsos conselhos editoriais ou em falsa prática de avaliação por pares, com o objetivo de obterem lucros com o pagamento das taxas de editoração. Nesse sentido, embora a busca pelo conhecimento e por respostas mais concretas intensifiquem as publicações dos dados científicos nas plataformas de preprints e/ou em revistas predatórias, estes não devem ser utilizados para orientar políticas de saúde ou a prática clínica. De modo semelhante, artigos disponíveis em bases de dados nacionais e internacionais devem ser avaliados quanto ao rigor, validade metodológica e qualidade dos dados obtidos.

Com base nas reflexões tecidas até o momento, algumas questões se renovam: na urgência de divulgar os resultados preliminares de estudos relacionados a COVID-19, os rigorosos métodos científicos estão sendo considerados? Estamos preparados "tecnicamente" para analisar um grande volume de estudos e lidar com novas informações a todo momento? Como identificar estudos com boa qualidade de evidência? Interpretações equivocadas podem se tornar armadilhas para os governantes, imprensa e sociedade, descredibilizando a ciência e colocando em risco a saúde da população. Apenas uma leitura crítica-reflexiva baseada em plausibilidade, coerência, consistência, relação temporal, força da associação, evidência experimental e analogia devem ser consideradas para fomentar o conhecimento sobre a COVID-19.

\section{Ciência, Tecnologia e Informação: Os Impactos Negativos da Desinformação}

A relação entre ciência e tecnologia pode ser avaliada por meio do conhecimento acumulado pela humanidade até a atualidade, e isto é particularmente verdade no mundo contemporâneo. No contexto da COVID-19, uma semana após a 
notificação dos primeiros casos de pneumonia por um agente biológico desconhecido, em janeiro de 2020, pesquisadores de Wuhan finalizaram o sequenciamento completo do genoma viral, disponibilizando-o no banco de dados internacional Global Initiative on Sharing All Influenza Data (GISAID) (Zhu et al., 2020). Após a confirmação do primeiro caso da COVID-19 no Brasil, uma parceria entre cientistas do Instituto Adolfo Lutz e das Universidade de São Paulo (USP) e de Oxford (Reino Unido) permitiu o sequenciamento do genoma do SARS-CoV-2 em apenas 48h (de Jesus et al., 2020). Nos dias atuais, a aprovação e o uso emergencial de sete vacinas ao redor do mundo, o desenvolvimento de respiradores simples e portáteis, o desenvolvimento, cada vez mais frequente, de aplicativos de rastreamento com o objetivo de geolocalizar os indivíduos infectados e os potenciais contatos extradomiciliares, são alguns poucos exemplos da inter-relação ciência e tecnologia, os quais têm contribuído de forma indiscutível no combate à COVID-19 (Altmann et al., 2020; Majid et al., 2020).

Os impactos dos avanços tecnológicos na ciência são inegáveis. Na pandemia da gripe espanhola em 1918, a descoberta do agente viral causador da doença só foi identificado treze anos após o término da pandemia (Goulart, 2020). A tecnologia permite explorar diferentes possibilidades, de forma mais rápida e fácil. Contudo, isso só é possível devido a evolução da tecnologia, uma vez que esta possibilita discutir evidências científicas, informações críticas e visões alternativas, mesmo que os sujeitos participantes estejam em diferentes regiões do planeta.

Paralelamente a estes benefícios, a tecnologia tornou a ciência acessível à sociedade, o que proporcionou não só o “fazer ciência" como o "divulgar a ciência”. Por conseguinte, a informação e a comunicação com a população e a imprensa tornaram-se estratégias fundamentais para o enfrentamento da COVID-19. Os números de casos confirmados e óbitos passaram a ser monitorados e disponibilizados em tempo real, a exemplo das plataformas online da JHU (https://coronavirus.jhu.edu/map.html), OMS (https://covid19.who.int) e, particularmente, no Brasil, da Rede CoVida, uma iniciativa do Centro de Integração de Dados e Conhecimentos para da Saúde (Cidacs/ Fiocruz Bahia) e da Universidade Federal da Bahia (https://painel.covid19br.org), todos dedicados à coleta e divulgação de dados relacionados à COVID-19. Além disso, foram criados meios para atendimento à população, como o aplicativo Coronavírus-SUS e o canal de atendimento por WhatsApp do Ministério da Saúde do Brasil (Oliveira et al., 2020), permitindo que as pessoas se apropriem dos saberes, participando de maneira mais ativa no processo de construção de conhecimento.

A grave crise sanitária que já ultrapassou o período de um ano deixa claro que a ciência depende direta ou indiretamente do avanço tecnológico, cujo impacto na sociedade a tornou uma das principais instituições sociais do século XXI. Por outro lado, o acesso rápido e fácil à informação tornou possível não só acompanhar o avanço da COVID-19 no país e no mundo, como também tem acelerado o acesso a um grande volume de informações incorretas e/ou imprecisas que são produzidas e compartilhadas por meio das mídias sociais. Na atual conjuntura, a divulgação dos achados científicos não se restringe à mídia jornalística ou aos meios oficiais de divulgação. Um estudo realizado por Gomes, Pena e Arroio (2020) mostrou que as mídias sociais de maior aderência são WhatsApp, Facebook, Instagram, Youtuber e Twitter, respectivamente, e que o WhatsApp é o aplicativo mais utilizado para compartilhar mensagens. Embora as mídias sociais representem uma oportunidade de democratização, em tempos de pandemia, a divulgação de recortes da realidade ou mesmo da falsificação da mesma, as chamadas fakes news, tem sido cada vez mais frequentes nas mídias sociais.

A manipulação deliberada ou interpretações inadequadas de evidências científicas têm sido utilizadas como forma de legitimar o negacionismo em relação à ciência, em especial no contexto do surgimento do SARS-CoV-2 ou do uso emergencial da vacina contra a COVID-19, produzindo percepções equivocadas da realidade da pandemia. Os movimentos anticiência e a divulgação de informações pseudocientíficas sempre existiram, mas, atualmente, têm ganhado um maior número de adeptos, muitas vezes, impulsionados pelas plataformas de mídias sociais e seus algoritmos que, em uma lógica mercadológica, potencializam a chegada de conteúdo conforme a preferência do usuário (Oliveira, 2020), fatos que contribuem para que o conhecimento científico possa ser ideologicamente manipulado. 
Um estudo realizado com estudantes universitários de uma instituição pública no Brasil mostrou que as teorias de conspiração são adotadas por pessoas com déficits perceptuais ou cognitivos, que rejeitam argumentos baseados no conhecimento científico e adotam discursos alternativos para substituir as evidências proporcionadas pela ciência, eventos tidos como ameaçadores ou desconhecidos (Rezende et al., 2019). Notícias como "o SARS-CoV-2 foi criado em laboratório", "no calor o SARS-CoV-2 não sobrevive”, “o uso prologando de máscara causa quadro de intoxicação e baixa oxigenação do organismo", "a vacina chinesa usa feto abortado ou transforma o paciente em homossexual” são algumas das inúmeras notícias falsas, associadas ao negacionismo científico e compartilhadas nas mídias sociais. A distorção da realidade causada pelas fake news e a velocidade de difusão proporcionada pelas mídias sociais têm dificultado o combate à COVID-19. Para Bynum (2018, p.340), “A ciência e tecnologia podem ser utilizadas com fins escusos com a mesma finalidade com que podem ser aplicadas ao bem comum. Precisamos de bons cientistas, mas também de bons cidadãos que assegurem que a nossa ciência torne o mundo um lugar melhor para todos".

Esses são alguns elementos que caracterizam dois cenários, de um lado o conhecimento produzido pela ciência e que deve ser aplicado em prol de uma sociedade que espera ansiosamente por uma resposta contra a COVID-19, do outro, um movimento sem precedentes questionando essa mesma ciência e disseminando soluções milagrosas não fundamentadas cientificamente. Dessa forma, discute-se sobre a necessidade de construir em todos os setores da sociedade, uma cultura crítica, especialmente da população leiga, para que a sociedade não fique refém de fake news e para que as falácias pseudocientíficas não representem uma ameaça ainda maior do que a COVID-19. No entanto, sabemos que estas questões esbarram em outras mais complexas, produto de aspectos históricos e sociais, sobretudo em fatores educacionais e de acesso à direitos fundamentais.

Nessa perspectiva, a divulgação científica também precisa ser um compromisso social, é preciso que o conhecimento sobre ciência chegue nas casas das pessoas com a mesma intensidade que chega à tecnologia, não apenas a informação sobre a cura de uma doença, mas, sobretudo, os caminhos que a ciência percorreu, os esforços empreendidos por pesquisadores, os investimentos e etapas necessárias para se chegar a um resultado esperado.

\section{A Ciência em Tempos de Crise: Entraves e Caminhos Possíveis}

Várias são as parcerias estabelecidas em todo o país e ao redor do mundo, permitindo acompanhar a evolução da pandemia da COVID-19 que já dura muitos meses. Embora a pandemia tenha despertado uma maior articulação científica, interesse e intercâmbio de conhecimento entre universidades, empresas farmacêuticas, instituições de pesquisa e unidades de saúde, ainda existem interesses divergentes que limitam o enfrentamento da COVID-19. A agilidade de países desenvolvidos no enfretamento de um desafio como esse mostra porque é importante manter um sistema sólido e internacionalizado de ciência, tecnologia e de formação de recursos humanos, o qual só pode ser garantido por políticas de longo prazo e continuidade de financiamento público.

A falta de compreensão sobre projeções epidemiológicas e, até mesmo, a utilização de estudos sem rigor científico têm embasado a flexibilização das medidas de distanciamento social. Não obstante, alguns líderes políticos têm na economia, uma das principais justificativas para a não aplicação dessa medida. O dilema infundado que tenta contrapor a saúde e economia tem sido estudado por diversas equipes de pesquisa. O conhecimento consensual indica que o arrefecimento da economia global seria uma realidade, mesmo que em menor escala, na hipótese de não aplicação de medidas de isolamento social. Pesquisadores da Universidade Northwestern, nos Estados Unidos, e da Free University, da Alemanha, desenvolveram modelos econômico-epidemiológicos para avaliar os possíveis impactos humanos e financeiros da COVID-19 nos Estados Unidos. Os autores sugerem que o enfrentamento da crise deve buscar padrões econômicos de equilíbrio entre custos e 
benefícios, e afirmam que em todos os cenários avaliados foi mais promissor introduzir o isolamento social (Eichenbaum; Rebelo; Trabandt, 2020).

Um caminho possível para que a ciência, seus métodos e percursos necessários cheguem à população, está na educação. Nesse campo, embora existam diferentes percursos possíveis, destacamos o letramento científico como uma estratégia de educação científica a médio e longo prazo, notadamente, nas séries iniciais de ensino. Esse é apenas um percurso possível, quando pensamos questões de acesso ao conhecimento científico. Fazer com que ele chegue ainda durante aos primeiros anos do processo de formação será de extrema importância, uma vez que o seu conceito está relacionado à "capacidade de se envolver com as questões relacionadas com a ciência e com a ideia da ciência, como um cidadão reflexivo" (OECD, 2015, p.7). Em outras palavras, o letramento configura-se como a capacidade de compreender e empregar o conhecimento científico, bem como pensar sob uma perspectiva científica. Ademais implica em despertar no sujeito a autonomia para tecer seu próprio ponto de vista, comprometido com os fatos e menos susceptíveis a emoções e crenças. Para Santos apud Shamos” (2007, p.479), "Um cidadão letrado não apenas sabe ler o vocabulário científico, mas é capaz de conversar, discutir, ler e escrever coerentemente em um contexto não-técnico, mas de forma significativa".

Em épocas passadas, o interesse em formar cientistas culminou na elaboração de projetos curriculares com ênfase na vivência do método científico nos Estados Unidos, durante a Guerra Fria no final dos anos de 1950, com o lançamento do primeiro satélite artificial - o Sputinik. Existia uma preocupação de preparar os jovens para adquirir uma postura de cientista, resultando na integração da educação científica na educação básica (Santos, 2007). Assim, uma educação que prepara a população para construir um conhecimento sobre o papel da ciência, não apenas do ponto de vista da resolução de um problema, mas da compreensão desta como uma prática social, contribuirá com a reorientação de cenários em que a ciência tem sido altamente "desconsiderada". De modo contrário, um modelo de ensino que não leve em consideração uma reflexão epistemológica da ciência estará longe de colaborar com o processo de alfabetização científica.

Conforme aborda Santos (2007, p.487), “o que se busca não é uma alfabetização em termos de propiciar somente a leitura de informações científicas e tecnológicas, mas a interpretação do seu papel social". No atual cenário da pandemia, surgem questionamentos sobre a relação indissociável entre ciência e educação, sobretudo em relação à formação para a reflexão e criticidade, e como isso tem sido e poderia ser aplicado ao ensino de ciências. Curiosamente, o projeto ROSE (The Relevance of Science Education) revelou que os jovens apresentam um alto interesse pela disciplina ciências, mas que gostam mais de outras disciplinas (Tonin; Tolentino-Neto; Ocampo, 2021). A lógica é simples, não se pode dar valor àquilo que não se conhece. Existe uma dificuldade em se comunicar a ciência para a população em geral e a distinção que tende a separar a academia de outras esferas sociais. Conforme Rezende et al. (2019), o negacionismo e/ou movimentos conspiratórios decorrem da incapacidade que os indivíduos têm de exercer um julgamento crítico a partir de dados científicos. Por fim, é necessário desenvolver a consciência científica por meio do letramento científico e/ou por mudanças curriculares no ensino fundamental e médio. Uma sociedade que valoriza a ciência apoia mais as iniciativas de pesquisa, participa mais de estudos que exigem participação voluntária e, por fim, avaliam com criticidade e propriedade as informações que recebem por meio das plataformas de mídias sociais.

\section{Considerações Finais}

O que fica pós-pandemia? Necessariamente, a questão do incentivo às inovações tecnológicas deve ser discutida em conjunto com o fomento à educação, letramento, pesquisa científica básica e aplicada. Assim, somente um trabalho em conjunto, no que se refere a ciência e tecnologia (C\&T), captação de recursos, investimento financeiro, colaboração de pesquisadores nacionais e internacionais com diferentes expertises, apoio e participação da sociedade civil e dos chefes de estado, permitirá avançar e finalizar com a pandemia da COVID-19. 
Esse conjunto de condições (causa) que, interagindo, produzem ciência (efeito/desfecho). Por sua vez, cabe aos pesquisadores e revisores garantir os critérios de rigor científico na divulgação dos resultados relacionados à pandemia da COVID-19. Assim como, cabe aos governantes, selecionar, analisar criticamente de forma sistematizada e racional, as inúmeras informações disponíveis na literatura, para que aquelas que representam o "conhecimento científico" possam subsidiar a formulação de políticas públicas e oferecer diretrizes para implementação de planos de enfretamento à COVID-19. Não obstante, é importante que mais estudos de natureza quantitativa e/ou qualitativa sobre a importância do método científico, sobretudo no contexto educativo na atualidade, bem como, acerca da disseminação e o impacto das fake news nas mídias sociais, sejam realizados a fim de aprofundar tais compreensões destacadas neste ensaio.

Quando a pandemia findar, indubitavelmente o perfil de mortalidade sofrerá grande impacto direta ou indiretamente por conta da COVID-19. O "novo normal” irá requerer mudanças no comportamento, no nível individual e comunitário. Mais estudos serão publicados e respostas com bases nos métodos científicos poderão ser dadas (ou não) frente a tantas incertezas e questionamentos sobre a COVID-19. Até lá, a utilização do conhecimento científico baseado em critérios técnicos e em evidências científicas - e não no achismo, ainda representará a melhor ferramenta para auxiliar os governantes na tomada de decisão no enfrentamento à COVID-19 ou a qualquer outra epidemia. E em um futuro próximo precisará ser destrinchada para que a população possa compreender melhor os caminhos traçados para obtenção daquele resultado.

\section{Referências}

Altmann, S. et al. (2020). Acceptability of App-Based Contact Tracing for COVID-19: Cross-Country Survey Study. JMIR Mhealth Uhealth, 8(8), e19857.

Beall, J. (2017). What I learned from predatory publishers. Biochem Med (Zagreb), 27(2), 273-278.

Brasil. (2020a). Ministério da Saúde. Secretaria de Vigilância em Saúde. Departamento de Análise em Saúde e Vigilância de Doenças Não Transmissíveis. Vigitel Brasil 2019: vigilância de fatores de risco e proteção para doenças crônicas por inquérito telefônico: estimativas sobre frequência e distribuição sociodemográfica de fatores de risco e proteção para doenças crônicas nas capitais dos 26 estados brasileiros e no Distrito Federal em 2019 . Brasília: Ministério da Saúde.

Brasil. (2020b). Ministério da Saúde. Secretaria de Vigilância em Saúde. Guia de Vigilância Epidemiológica Emergência de Saúde Pública de Importância Nacional pela Doença pelo Coronavírus 2019: Vigilância de Síndromes Respiratórias Agudas COVID-19, 05 de agosto de 2020. Brasília: Ministério da Saúde.

Bynum, W. (2018). Uma breve história da ciência. L\&PM.

Chalmers, A. F. (1993). O que é ciências, afinal? (1a ed.), Brasiliense.

Conceição, V. A. dos S. \& Chagas, A. M. (2020). O pesquisador e a divulgação científica em contexto de cibercultura e inteligência artificial. Acta Scientiarum Education, 42, e52879.

Dal-Fabbro, A. L. (2011). Causalidade em epidemiologia. In: Franco, L. J.; Passos, A. D. C. Fundamentos De Epidemiologia. Manole.

Dantas, L. F. S. \& Deccache-Maia, E. (2020). Divulgação Científica no combate às Fake News em tempos de Covid-19. Research, Society and Development, 9(7), e797974776.

de Jesus, J. G. et al. (2020). First cases of coronavirus disease (COVID-19) in Brazil, South America (2 genomes, 3rd March 2020). Virological.

Eichenbaum, M. S., Rebelo, S. \& Trabandt, M. (2020). The Macroeconomics of Epidemics. Report No.: 26882. [citado em 2021 mar. 23]. Disponível em: https://www.nber.org/papers/w26882

Elm, U. et al. (2020). Cyllage City COVID-19 Outbreak Linked to Zubat Consumption. Am J Biomed Sci, 8(2), 140-42.

Francelin, M. M. (2004). Ciência, senso comum e revoluções científicas: ressonâncias e paradoxos. Ciência da Informação, 33(3), $26-34$.

Gandhi, M., Yokoe, D. S. \& Havlir, D. V. (2020) Asymptomatic Transmission, the Achilles' Heel of Current Strategies to Control Covid-19. N Engl J Med, e2009758.

Gautret, P. et al. (2020). Hydroxychloroquine and azithromycin as a treatment of COVID-19: results of an open-label non-randomized clinical trial. Int $J$ Antimicrob Agents, 20, 105949.

Gomes, S. F., Penna, J. C. B. de O. \& Arroio, A. (2020) Fake News Científicas: Percepção, Persuasão e Letramento. Ciênc. educ. (Bauru), 26, e20018, 2020. Goulart, A. C. (2005) Revisitando a espanhola: a gripe pandêmica de 1918 no Rio de Janeiro. Hist Cienc Saude-Manguinhos, 12(1), 101-42.

Inloco [Internet]. 2021. Geolocalização e privacidade: os dados no combate à COVID-19 [cited 2021 Abr 1]. https://www.inloco.com.br/covid-19 
Johns Hopkins University (JHU). (2021). Covid-19 dashboard by the center for systems science and engineering (CSSE) at Johns Hopkins university. Baltimore: Johns Hopkins University; 2020. [citado em 2021 abr. 1]. https://coronavirus.jhu.edu/map.html

Jones, N. R. et al. (2020). Two metres or one: what is the evidence for physical distancing in covid-19? BMJ, $370, \mathrm{~m} 3223$.

Leite, F. T. (2008). Metodologia científica: métodos e técnicas de pesquisa (monografias, dissertaçõteses e livros). (2a ed.), Ideias \& Letras.

Mahévas, M. et al. (2020). Clinical efficacy of hydroxychloroquine in patients with covid-19 pneumonia who require oxygen: observational comparative study using routine care data. $B M J, 369, \mathrm{~m} 1844$.

Majid, B. E. et al. (2020). Preliminary design of an innovative, simple, and easy-to-build portable ventilator for COVID-19 patients. EuroMediterr J Environ Integr, 5(2), 23.

Meneghetti, F. K. (2011). O que é um ensaio-teórico?. Revista de Administração Contemporânea, 15(2), $320-332$.

Novaes, H. M. D. \& Carvalheiro, J. da R. (2007). Ciência, tecnologia e inovação em saúde e desenvolvimento social e qualidade de vida: teses para debate. Ciênc. saúde coletiva, 12, 1841-1849.

OECD. (2015). Programa Internacional de Avaliação de Estudantes (PISA). Matriz de Avaliação de Ciências. Resumo do Documento: PISA 2015 Science $\begin{array}{lllll}\text { Framework } & \text { (2013). } & \text { em } & \text { 2021 }\end{array}$ http://download.inep.gov.br/acoes_internacionais/pisa/marcos_referenciais/2015/matriz_de_ciencias_PISA_2015.pdf

Oliveira, T. (2020). Desinformação científica em tempos de crise epistêmica: circulação de teorias da conspiração nas plataformas de mídias sociais. Fronteiras, 22(1), 2020.

Oliveira, W. K. et al. (2020). Como o Brasil pode deter a COVID-19. Epidemiol Serv Saúde, 29(2), e2020044.

Oreskes, N. (2019). Why Trust Science? Princeton University Press.

Palácio, M. A. V. \& Takenami, I. (2020). Em tempos de pandemia pela COVID-19: o desafio para a educação em saúde. Vigilância Sanitária Em Debate: Sociedade, Ciência \& Tecnologia, 8(2), 10-15.

Popper, K. R. (1972). A lógica da pesquisa científica. (3a ed.), Cultrix.

Rezende, A. T. et al. (2019). Teorias da conspiração: significados em contexto brasileiro. Estud. Psicol, 36, e180010.

Santos, W. L. P. (2007). Educação científica na perspectiva de letramento como prática social: funções, princípios e desafios. Revista Brasileira de Educação, 12(36), 474-550.

Selvaraj, V., Herman, K. \& Dapaah-Afriyie, K. (2020). Severe, Symptomatic Reinfection in a Patient with COVID-19. R I Med J, 103(10), 24-26.

Sheldon, T. (2018). Preprints could promote confusion and distortion. Nature, 559:445.

Szklo, M. (2006). Quality of scientific articles. Revista de Saúde Pública, 40, 30-35.

Tang, W. et al. (2020). Hydroxychloroquine in patients with mainly mild to moderate coronavirus disease 2019: open label, randomised controlled trial. BMJ, $369, \mathrm{~m} 1849$.

Tonin, K. G., Tolentino-Neto, L. C. B. de, \& Ocampo, D. M. (2021). Os jovens brasileiros e a sua preferência pela disciplina Ciências. Research, Society and Development, 10(5), e8210514549.

Visoni, R. M. \& Canalle, J. B. G. (2009). Bartolomeu Lourenço de Gusmão: o primeiro cientista brasileiro. Revista Brasileira de Ensino de Física, 31(3), 3604.1-3604.12.

Volpato, G. (2008). Publicação Cientifica. (3a ed.), Cultura Acadêmica.

Wilder-Smith, A. \& Freedman, D. O. (2020). Isolation, quarantine, social distancing and community containment: pivotal role for old-style public health measures in the novel coronavirus (2019-nCoV) outbreak. J Travel Med, 27(2), 1-4.

World Health Organization (WHOa). WHO Director-General's Opening Remarks at the Media Briefing on COVID-19-11 March 2020. Geneva: WHO; 2020a. [citado em 2021 mar. 21]. https://www.who.int/dg/speeches/detail/who-director-general-s-opening-remarks-at-the-media-briefing-on-covid-19---11march-2020

World Health Organization (WHOb). 2019 Novel Coronavirus (2019-nCoV): Strategic preparedness and response plan. Geneva: World Health Organization; 2020c. [citado em 2021 mar. 21]. https://www.who.int/docs/default-source/coronaviruse/srp-04022020.pdf?ua=1

World Health Organization (WHOc). COVID-19 Strategic Preparedness and Response Plan - Operational Planning Guidelines to Support Country Preparedness and Response. Geneva: World Health Organization; 2020b. [citado em 2021 mar. 21]. https://www.who.int/docs/defaultsource/coronaviruse/covid-19-sprp-unct-guidelines.pdf

World Health Organization (WHOd). Considerations for quarantine of individuals in the context of containment for coronavirus disease (COVID-19). Geneva: World Health Organization; 2020d. [citado em 2021 mar. 21]. https://www.who.int/publications/i/item/considerations-for-quarantine-of-individualsin-the-context-of-containment-for-coronavirus-disease-(covid-19)

Wu, F. et al. (2020). A new coronavirus associated with human respiratory disease in China. Nature, 579, $265-269$.

Zhu, N. et al. (2020). A novel coronavirus from patients with pneumonia in China, 2019. N Engl J Med, $382(8), 727-33$. 